\title{
Comprehensive behavioral phenotyping of ryanodine receptor type 3 (RyR3) knockout mice: decreased social contact duration in two social interaction tests
}

\section{Naoki Matsuo ${ }^{1,2 \dagger}$, Koichi Tanda ${ }^{3,4 t}$, Kazuo Nakanishi ${ }^{3}$, Nobuyuki Yamasaki, ${ }^{3,5}$, Keiko Toyama ${ }^{1,2}$, Keizo Takao ${ }^{1,2,3,6}$, Hiroshi Takeshima ${ }^{7}$ and Tsuyoshi Miyakawa ${ }^{1,2,3,6 *}$}

\author{
Division of Systems Medical Science, Institute for Comprehensive Medical Science, Fujita Health University, Toyoake, Japan \\ 2 Core Research for Evolutional Science and Technology (CREST), Japan Science and Technology Agency, Kawaguchi, Japan \\ 3 Genetic Engineering and Functional Genomics Group, Frontier Technology Center, Graduate School of Medicine, Kyoto University, Kyoto, Japan \\ 4 Department of Pediatrics, Kyoto Prefectural University of Medicine, Kyoto, Japan \\ 5 Department of Psychiatry, Graduate School of Medicine, Kyoto University, Kyoto, Japan \\ ${ }_{6}$ Institute for Bioinformatics Research and Development (BIRD), Japan Science and Technology Agency, Kawaguchi, Japan \\ 7 Department of Biological Chemistry, Graduate School of Pharmaceutical Sciences, Kyoto University, Kyoto, Japan
}

\section{Edited by:}

Shigeyoshi Itohara, RIKEN Brain

Science Institute, Japan

\section{Reviewed by:}

Craig M. Powell, Department of

Neurology, The University of Texas

Southwestern Medical Centre, USA

Satoshi Kida, Tokyo University of

Agriculture and Technology, Japan

\section{*Correspondence:}

Tsuyoshi Miyakawa, Division of

Systems Medical Science, Institute for

Comprehensive Medical Science,

Fujita Health University, 1-98

Dengakugakubo, Kutsukake, Toyoake,

Aichi 470-1192, Japan.

e-mail:miyakawa@fujita-hu.ac.jp

${ }^{\dagger}$ Naoki Matsuo and Koichi Tanda have

contributed equally to this work
Dynamic regulation of the intracellular $\mathrm{Ca}^{2+}$ concentration is crucial for various neuronal functions such as synaptic transmission and plasticity, and gene expression. Ryanodine receptors (RyRs) are a family of intracellular calcium release channels that mediate calcium-induced calcium release from the endoplasmic reticulum. Among the three RyR isoforms, RyR3 is preferentially expressed in the brain especially in the hippocampus and striatum. To investigate the behavioral effects of RyR3 deficiency, we subjected RyR3 knockout (RyR3-/-) mice to a battery of behavioral tests. RyR3-/- mice exhibited significantly decreased social contact duration in two different social interaction tests, where two mice can freely move and make contacts with each other. They also exhibited hyperactivity and mildly impaired prepulse inhibition and latent inhibition while they did not show significant abnormalities in motor function and working and reference memory tests. These results indicate that RyR3 has an important role in locomotor activity and social behavior.

Keywords: ryanodine receptor, knockout mouse, behavior, social interaction, schizophrenia, autism

\section{INTRODUCTION}

Establishing animal models of psychiatric disorders by utilizing genetically engineered mice is essential for investigating the pathogenesis/pathophysiology and treatment of these disorders (Arguello and Gogos, 2006; Chen et al., 2006; Gainetdinov et al., 2001; Powell and Miyakawa, 2006). Previously, we reported that forebrainspecific calcineurin (also called as protein phosphatase $2 \mathrm{~B}$ ) knockout mice have severe working/episodic-like memory deficits (Zeng et al., 2001), and exhibit a spectrum of abnormal behaviors related to schizophrenia in humans (Miyakawa et al., 2003). In addition, we and other groups have identified the PPP3CC gene, which encodes the calcineurin gamma subunit, as a potential schizophrenia and bipolar disorder susceptibility gene (Gerber et al., 2003; Horiuchi et al., 2007; Liu et al., 2007; Mathieu et al., 2008; Shi et al., 2008; Yamada et al., 2007). These studies strongly demonstrated the usefulness of applying a comprehensive battery of behavioral test on genetically engineered mice to efficiently obtain a mouse model of human psychiatric disorders. Thus, we have been applying this approach to various strains of mice bearing mutations of the genes encoding the molecules related to either the calcineurin signaling pathways or calcineurin-related neural mechanisms (Arron et al., 2006; Takao and Miyakawa, 2006a; Takao et al., 2007; Yamasaki et al., 2008). Here we focused on the brain-type ryanodine receptor
(RyR3), one of the molecules that form a macromolecular signaling complex with calcineurin (Snyder et al., 1998).

Spatially and temporally regulated changes of the intracellular calcium concentration $\left[\mathrm{Ca}^{2+}\right]_{\mathrm{i}}$ plays a variety of critical roles in numerous cell types. For example, elevated $\left[\mathrm{Ca}^{2+}\right]_{\mathrm{i}}$ controls muscle contraction in skeletal and cardiac muscles, and activity-dependent gene expression, synaptic transmission and plasticity in neurons. $\left[\mathrm{Ca}^{2+}\right]_{i}$ can be increased either by the opening of calcium channels on the plasma membrane, to generate an influx of $\mathrm{Ca}^{2+}$ from the extracellular fluid, or by the activation of $\mathrm{Ca}^{2+}$ release channels located on the endoplasmic and sarcoplasmic reticula. Two different families of calcium release channels located on these intracellular $\mathrm{Ca}^{2+}$ stores have been identified: the inositol 1,4,5-triphosphate receptor (IP3R) (Ferris and Snyder, 1992; Mikoshiba, 1993); and the ryanodine receptor (RyR) (McPherson and Campbell, 1993; Sorrentino and Volpe, 1993). In neurons, RyR acts as a calciuminduced calcium release channel and its activity is enhanced by increased $\left[\mathrm{Ca}^{2+}\right]_{\mathrm{i}}$ through the voltage-dependent calcium channels or $\mathrm{N}$-methyl-D-aspartate receptors on the plasma membrane (Bardo et al., 2006; Berridge, 1998; Rose and Konnerth, 2001). On the other hand, calcium release through IP3R is triggered by inositol 1,4,5-triphosphate, a metabolic product of phospholipase $\mathrm{C}$ activity, which is coupled to the activation of metabotropic glutamate 
receptors at the excitatory synapses (Bardo et al., 2006; Berridge, 1998; Rose and Konnerth, 2001). The distinct signaling pathways and heterogeneous neuronal distribution of these two types of channels are likely to contribute to the regulated release of intracellular $\mathrm{Ca}^{2+}$ in a spatial- and temporal-dependent manner.

The RyRs are $\sim 2200 \mathrm{kDa}$ homotetrameric complexes and interact with regulatory proteins such as calmodulin, CaMKII, FK506binding protein 12 (FKBP12 or calstabin), and calcineurin to control channel activity in response to extracellular stimuli, and thereby regulates intracellular calcium release (Snyder et al., 1998; Zalk et al., 2007). For example, dissociation of the calcineurinFKBP12 complex from RyRs leads to an increased open channel probability and induces subconductance states (Brillantes et al., 1994), suggesting that the complex stabilizes RyR and prevents calcium leakage.

The RyR family has three isoforms encoded by three distinct genes and termed according to their tissue-type preference pattern of expression; skeletal type (RyR1), cardiac type (RyR2), and brain type (RyR3). Although all isoforms are expressed in the brain, they exhibit differential expression patterns. In the adult mouse brain, RyR1 is predominantly expressed in cerebellar Purkinje cells and the dentate gyrus of the hippocampus. RyR2 is broadly expressed in most brain regions. RyR3 is predominantly expressed in the hippocampus, and striatum (Giannini et al., 1995; Kuwajima et al., 1992; Mori et al., 2000). The functional significance of each isoform has been examined using mice with a targeted disruption. The RyR1 knockout mice died perinatally with gross abnormalities of skeletal muscle (Takeshima et al., 1994). Mutant mice lacking RyR2 also died at approximately embryonic day 10 with morphologic abnormalities in the heart tube (Takeshima et al., 1998). In contrast, the RyR3 knockout mice were fertile and displayed no gross abnormalities (Bertocchini et al., 1997; Futatsugi et al., 1999; Takeshima et al., 1996). However, RyR3-/- mice exhibited increased locomotor activity, suggesting abnormalities in certain neurons (Takeshima et al., 1996). Furthermore, spatial learning was altered in RyR3-/- mice (Balschun et al., 1999; Futatsugi et al., 1999). The RyR3-/- mice also exhibited impairments in contextual fear conditioning, passive avoidance, and Y-maze tests (Kouzu et al., 2000). Consistent with the behavioral changes associated with the hippocampus, altered long-term potentiation and depression were detected in the hippocampus of RyR3-/- mice (Balschun et al., 1999; Futatsugi et al., 1999; Shimuta et al., 2001). Attenuation of ryanodine-induced dopamine release was also reported in the striatal slices of RyR3-/- mice, suggesting an involvement of RyR3 in dopamine release in striatal neurons (Wan et al., 1999). In human, RyR3 gene is located at 15q14-15 (Sorrentino et al., 1993), that was suggested as a putative schizophrenia (Leonard and Freedman, 2006; Liu et al., 2001; Moon et al., 2006; Williams et al., 1999) and autism (Abrahams and Geschwind, 2008) susceptibility locus.

To assess the possible utility of RyR3-/- mice as an animal model of psychiatric disorders, we subjected them to a comprehensive behavioral test battery (Takao and Miyakawa, 2006a; Takao et al., 2007). We found that RyR3-/- mice show hyperactivity, decreased social interaction and mildly impaired prepulse inhibition and latent inhibition; while they did not show significant deficits in motor function and working and reference memory tasks. It is noteworthy that the lack of one of the three isoforms of RyRs, all of which are redundantly expressed in the brain, results in such significant behavioral abnormalities. This implies a critical function for RyRs in regulating the $\left[\mathrm{Ca}^{2+}\right]_{\mathrm{i}}$ in response to various extracellular signals in the mammalian brain in vivo.

\section{MATERIALS AND METHODS ANIMALS AND EXPERIMENTAL DESIGN}

RyR3 knockout mice were generated as previously described (Takeshima et al., 1996). They were bred for at least five generations on the C57BL/6 background. All behavioral tests were carried out with male mice that were 9 weeks old at the start of testing. Mice were housed in a room with a 12-h light/dark cycle (lights on at 7:00 a.m.) with access to food and water ad libitum. Behavioral testing was performed between 9:00 a.m. and 6:00 p.m. After the tests, all apparatus was cleaned with diluted sodium hypochlorite solution to prevent a bias due to olfactory cues. All behavioral tests were conducted in a manner similar to those previously described (Miyakawa et al., 2003; Yamasaki et al., 2008). The raw data of behavioral tests, which are not described in this paper, are disclosed in the gene-brain-phenotyping database (https://behav.hmro.med. kyoto-u.ac.jp/). All behavioral testing procedures were approved by the Animal Care and Use Committee of Kyoto University Graduate School of Medicine.

\section{BEHAVIORAL TESTS}

\section{Neurological screen}

The righting, whisker touch, and ear twitch reflexes were evaluated. A number of physical features, including the presence of whiskers or bald hair patches, were also recorded.

\section{Neuromuscular strength}

Neuromuscular strength was tested with the grip strength and wire hang tests. A grip strength meter (O'Hara \& Co., Tokyo, Japan) was used to assess forelimb grip strength. Mice were lifted and held by their tail so that their forepaws could grasp a wire grid. The mice were then gently pulled backward by the tail with their posture parallel to the surface of the table until they released the grid. The peak force applied by the forelimbs of the mouse was recorded in Newton (N). Each mouse was tested three times, and the greatest measured value was used for statistical analysis. In the wire hang test, the mouse was placed on a wire mesh that was then inverted and waved gently, so that the mouse gripped the wire. Latency to fall was recorded, with a 60-s cut-off time.

\section{Hot plate test}

The hot plate test was used to evaluate sensitivity to a painful stimulus. Mice were placed on a $55.0( \pm 0.3)^{\circ} \mathrm{C}$ hot plate (Columbus Instruments), and latency to the first hind-paw response was recorded. The hind-paw response was defined as either a foot shake or a paw lick.

\section{Rotarod test}

The rotarod test, using an accelerating rotarod (UGO Basile Accelerating Rotarod), was performed by placing mice on rotating drums ( $3 \mathrm{~cm}$ diameter) and measuring the time each animal was able to maintain its balance on the rod. The speed of the rotarod accelerated from 4 to $40 \mathrm{rpm}$ over a 5 -min period. 


\section{Open field test}

Each mouse was placed in the center of the open field apparatus $(40 \mathrm{~cm} \times 40 \mathrm{~cm} \times 30 \mathrm{~cm}$; Accuscan Instruments, Columbus, $\mathrm{OH}$, USA). Total distance traveled (in centimeter), vertical activity (rearing measured by counting the number of photobeam interruptions), time spent in the center, the beam-break counts for stereotyped behaviors, and number of fecal boli were recorded. Data were collected for $120 \mathrm{~min}$.

\section{Light/dark transition test}

Light/dark transition test was conducted as previously described (Takao and Miyakawa, 2006b). The apparatus used for the light/ dark transition test consisted of a cage $(21 \mathrm{~cm} \times 42 \mathrm{~cm} \times 25 \mathrm{~cm})$ divided into two sections of equal size by a partition containing a door (O'Hara \& Co., Tokyo, Japan). One chamber was brightly illuminated $(390 \mathrm{~lx})$, whereas the other chamber was dark $(2 \mathrm{~lx})$. Mice were placed into the dark side and allowed to move freely between the two chambers with the door open for $10 \mathrm{~min}$. The total number of transitions between chambers, time spent in each side, first latency to enter the light side and distance traveled were recorded automatically.

\section{Elevated plus-maze test}

Elevated plus-maze test was conducted as previously described (Komada et al., 2008). The elevated plus-maze (O'Hara \& Co., Tokyo, Japan $)$ consisted of two open arms $(25 \mathrm{~cm} \times 5 \mathrm{~cm})$ and two enclosed arms of the same size, with $15 \mathrm{~cm}$ high transparent walls. The arms and central square were made of white plastic plates and were elevated to a height of $55 \mathrm{~cm}$ above the floor. To minimize the likelihood of animals falling from the apparatus, $3 \mathrm{~mm}$ high plastic ledges were provided for the open arms. Arms of the same type were arranged at opposite sides to each other. Each mouse was placed in the central square of the maze $(5 \mathrm{~cm} \times 5 \mathrm{~cm})$, facing one of the closed arms. Mouse behavior was recorded during a 10 -min test period. The number of entries into, and the time spent in the open and enclosed arms, were recorded. For data analysis, we used the following four measures: the percentage of entries into the open arms, the time spent in the open arms (seconds), the number of total entries, and total distance traveled (centimeter). Data acquisition and analysis were performed automatically using Image EP software.

\section{Porsolt forced swim test}

The apparatus consisted of four plastic cylinders $(20 \mathrm{~cm}$ height $\times 10 \mathrm{~cm}$ diameter). The cylinders were filled with water $\left(23^{\circ} \mathrm{C}\right)$ up to a height of $7.5 \mathrm{~cm}$. Mice were placed into the cylinders, and their behavior recorded over a 10 -min test period. Data acquisition and analysis were performed automatically, using Image PS software (see Section 'IMAGE ANALYSIS'). Distance traveled was measured by Image OF software (see Section 'IMAGE ANALYSIS') using stored image files.

\section{Social interaction test in a novel environment}

Two mice of identical genotypes that were previously housed in different cages, were placed into a box together $(40 \mathrm{~cm} \times 40 \mathrm{~cm} \times 30 \mathrm{~cm})$ and allowed to explore freely for $10 \mathrm{~min}$. Social behavior was monitored by a CCD camera, which was connected to a Macintosh computer. Analysis was performed automatically using Image SI software. The total duration of contacts, the number of contacts, the number of active contacts, mean duration per contact, and total distance traveled were measured. The number of active contacts was defined as follows. Images were captured at one frame per second, and the distance traveled between two successive frames was calculated for each mouse. If the two mice contacted each other and the distance traveled by either mouse was longer than $5 \mathrm{~cm}$, the behavior was considered as an 'active contact'.

\section{Locomotor activity monitoring in the home cage}

A system that automatically analyzes the locomotor activity of mice in their home cage was used. The system contains a home cage $(29 \mathrm{~cm} \times 18 \mathrm{~cm} \times 12 \mathrm{~cm})$ and a filtered cage top, separated by a $13-\mathrm{cm}$-high metal stand containing an infrared video camera, which is attached to the top of the stand. Each mouse was individually housed in each home cage, and their locomotor activity was monitored for at least 5 days. Outputs from the video cameras were fed into a Macintosh computer. Images from each cage were captured at a rate of one frame per second, and distance traveled was measured automatically using Image HA software (see Section 'IMAGE ANALYSIS').

\section{Crawley's sociability and social novelty preference test}

Crawley's sociability and social novelty preference test is welldesigned method to investigate the complex genetics of social behaviors. The social testing apparatus consisted of a rectangular, three-chambered box and a lid with an infrared video camera (Ohara \& Co., Tokyo). Each chamber was $20 \mathrm{~cm} \times 40 \mathrm{~cm} \times 22 \mathrm{~cm}$ and the dividing walls were made from clear Plexiglas, with small square openings $(5 \mathrm{~cm} \times 3 \mathrm{~cm}$ ) allowing access into each chamber. An unfamiliar C57BL/6J male (stranger 1), that had had no prior contact with the subject mice, was placed in one of the side chambers. The location of stranger 1 in the left vs. right side chamber was systematically alternated between trials. The stranger mouse was enclosed in a small, round wire cage, which allowed nose contact between the bars, but prevented fighting. The cage was $11 \mathrm{~cm}$ in height, with a bottom diameter of $9 \mathrm{~cm}$, vertical bars $0.5 \mathrm{~cm}$, and horizontal bars spaced $1 \mathrm{~cm}$ apart. The subject mouse was first placed in the middle chamber and allowed to explore the entire social test box for a 10-min session. The amount of time spent in each chamber was measured with the aid of a camera fitted on top of the box. Each mouse was tested in a 10-min session to quantify social preference for the first stranger. After the first 10-min session, a second unfamiliar mouse was placed in the chamber that had been empty during the first 10-min session. This second stranger was also enclosed in an identical small wire cage. The test mouse thus had a choice between the first, already-investigated unfamiliar mouse (stranger 1), and the novel unfamiliar mouse (stranger 2). The amount of time spent in each chamber during the second 10-min was measured as described above. Data acquisition and analysis were performed automatically, using Image J based original program (Image CSI: see 'IMAGE ANALYSIS') software.

\section{Startle response/prepulse inhibition tests}

A startle reflex measurement system (O'Hara \& Co., Tokyo, Japan) was used to measure startle response and prepulse inhibition. A test 
session began by placing a mouse in a plastic cylinder where it was left undisturbed for $10 \mathrm{~min}$. White noise $(40 \mathrm{~ms})$ was used as the startle stimulus for all trial types. The startle response was recorded for $140 \mathrm{~ms}$ (measuring the response every $1 \mathrm{~ms}$ ) starting with the onset of the prepulse stimulus. The background noise level in each chamber was $70 \mathrm{~dB}$. The peak startle amplitude recorded during the $140 \mathrm{~ms}$ sampling window was used as the dependent variable. A test session consisted of six trial types (i.e., two types for startle stimulus only trials, and four types for prepulse inhibition trials). The intensity of the startle stimulus was 110 or $120 \mathrm{~dB}$. The prepulse sound was presented $100 \mathrm{~ms}$ before the startle stimulus, and its intensity was 74 or $78 \mathrm{~dB}$. Four combinations of prepulse and startle stimuli were used (74-110, 78-110, 74-120, and 78-120 dB). Six blocks of the six trial types were presented in pseudorandom order such that each trial type was presented once within a block. The average inter-trial interval was $15 \mathrm{~s}$ (range 10-20 s).

\section{Latent inhibition test}

On the first day, each mouse was placed in a conditioning chamber (O'hara \& Co., Tokyo). The mice were divided into two groups: preexposed (P) group and non pre-exposed (NP) group. The P group received 40 white noise tones ( $55 \mathrm{~dB}, 5 \mathrm{~s}$ duration, 25 s interstimulus interval), whereas the NP group received no stimulus during an equivalent period. Immediately after the tone pre-exposure or the exposure to the chamber, tone-shock pairs consisting of a 5-s tone coterminating with a 2 -s foot shock at $0.25 \mathrm{~mA}$ were delivered to both groups with a 25 -s interstimulus interval. Afterward, mice remained in the chamber for $25 \mathrm{~s}$ before being returned to the home cage. On day 2, the mice were placed back in the conditioning chamber for $5 \mathrm{~min}$ for the measurement of freezing to the context. On the same day, the mice were put in a triangular box $(35 \mathrm{~cm} \times 35 \mathrm{~cm} \times 40 \mathrm{~cm})$ made of white opaque Plexiglas, which was located in a different room, and after $180 \mathrm{~s}$, a 180-s tone was delivered to measure cued freezing.

\section{Eight-arm radial maze test}

Fully automated eight-arm radial maze apparatuses (O'Hara \& Co., Tokyo, Japan) were used. The floor of the maze was made of white plastic, and the wall $(25 \mathrm{~cm}$ high) consisted of transparent plastic. Each arm $(9 \mathrm{~cm} \times 40 \mathrm{~cm})$ radiated from an octagonal central starting platform (perimeter $12 \mathrm{~cm} \times 8 \mathrm{~cm}$ ) like the spokes of a wheel. Identical food wells ( $1.4 \mathrm{~cm}$ deep and $1.4 \mathrm{~cm}$ in diameter) with pellet sensors were placed at the distal end of each arm. The pellet sensors were able to automatically record pellet intake by the mice. The maze was elevated $75 \mathrm{~cm}$ above the floor and placed in a dimly-lit room with several extra-maze cues. During the experiment, the maze was maintained in a constant orientation. One week before pretraining, animals were deprived of food until their body weight was reduced to 80 to $85 \%$ of the initial level. Pretraining started on the eighth day. Each mouse was placed in the central starting platform and allowed to explore and consume food pellets scattered throughout the whole maze for a 30-min period (one session per mouse). After completion of the initial pretraining, mice received further pretraining to take a food pellet from each food well after being placed at the distal end of each arm. A trial was finished after the mouse consumed the pellet. This was repeated eight times, using eight different arms, for each mouse. After these pretraining trials, actual maze acquisition trials were performed. In the spatial working memory task of the eight-arm radial maze, all eight arms were baited with food pellets. Mice were placed on the central platform and allowed to obtain all eight pellets within $25 \mathrm{~min}$. A trial was terminated immediately after all eight pellets were consumed or $25 \mathrm{~min}$ had elapsed. An 'arm visit' was defined as traveling more than $5 \mathrm{~cm}$ from the central platform. The mice were confined at the center platform for $5 \mathrm{~s}$ after each arm choice. The animals went through one trial per day. For each trial, arm choice, latency to obtain all pellets, distance traveled, number of different arms chosen within the first eight choices, the number of revisiting, and omission errors were automatically recorded.

In the reference memory task of the eight-arm radial maze, one of the eight arms was consistently baited with one food pellet in the food well and a trial was terminated immediately after the pellet was consumed. Data acquisition, control of guillotine doors, and data analysis were performed by Image RM software (see Section 'IMAGE ANALYSIS').

\section{IMAGE ANALYSIS}

The applications used for the behavioral studies (Image LD, Image EP, Image RM, Image FZ, Image SI, and Image HA) were based on the public domain NIH Image program (developed at the U.S. National Institutes of Health and available on the Internet at http://rsb.info. nih.gov/nih-image/) and ImageJ program (http://rsb.info.nih.gov/ ij/), which were modified for each test by Tsuyoshi Miyakawa (available through O’Hara \& Co., Tokyo, Japan).

\section{STATISTICAL ANALYSIS}

Statistical analysis was conducted using StatView (SAS Institute, Cary, NC, USA). Data were analyzed by two-way ANOVA, or two-way repeated measures ANOVA, unless noted otherwise. Values in graphs were expressed as mean \pm SEM.

\section{RESULTS}

\section{LOCOMOTOR ACTIVITY AND ANXIETY- AND DEPRESSION-LIKE} BEHAVIORS IN RyR3-I- MICE

To address the behavioral effects of RyR3 deficiency, we subjected RyR3-/- mice and their wild-type littermates to a comprehensive battery of behavioral tests (Takao and Miyakawa, 2006a; Takao et al., 2007). RyR3-/- mice appeared healthy and showed no obvious differences in physical characteristics, with the exception of a slight decrease in body weight relative to wild-type mice (Figure 1A; $F_{1,101}=5.397, p=0.0224$ ). There were no significant differences in body temperature (Figure 1B; $F_{1,101}=0.001$, $p=0.9808$ ), neuromuscular strength (wire hang and grip strength tests) (Figures 1C,D; $F_{1,101}=0.239, p=0.6257$, and $F_{1,101}=0.842$, $p=0.3610$, respectively), sensitivity to a painful stimulus (hot plate test) (Figure 1E; $F_{1,45}=2.717, p=0.1062$ ), or motor functions (rotarod test) (Figure 1F; $F_{1,45}=0.243, p=0.6246$ ) between the wild-type and the knockout mice.

Examination of the locomotor activity of RyR3-/- mice in several behavioral tasks consistently revealed a hyperlocomotor activity phenotype. Total distance traveled by RyR3-/- mice was significantly greater than that of controls during an open field test (Figure 2A; $F_{1,158}=18.945, p<0.0001$ ), light-dark transition test (Figure 3A; dark: $F_{1,171}=69.155$, light: $F_{1,171}=18.083, p<0.0001$ ), elevated plus-maze test (Figure 4A; $F_{1,83}=8.864, p=0.0038$ ), and the social 
A

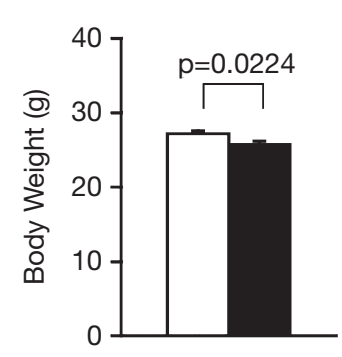

C

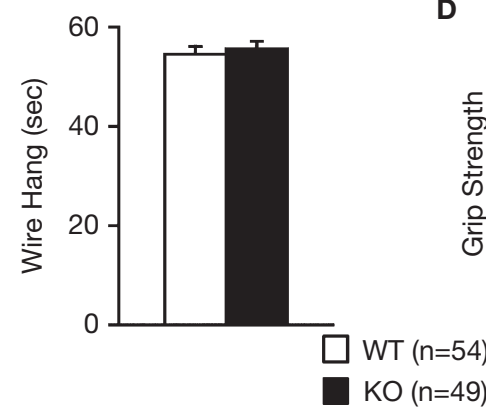

B
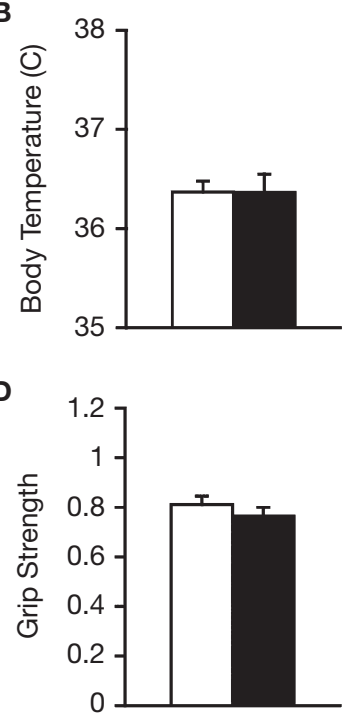

E

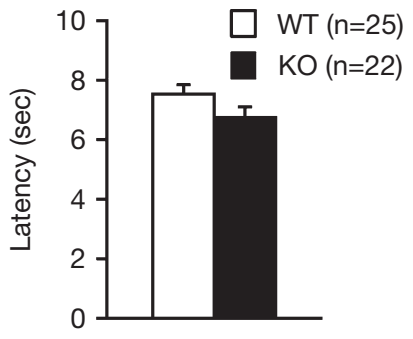

F

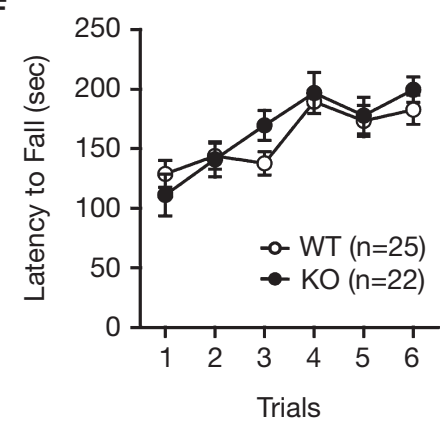

FIGURE 1 | Normal physical characteristics of RyR3-I- mice. (A) Body weight. (B) Body temperature. (C) Wire hang test. (D) Grip strength test. (E) Hot plate test. (F) Rotarod test.
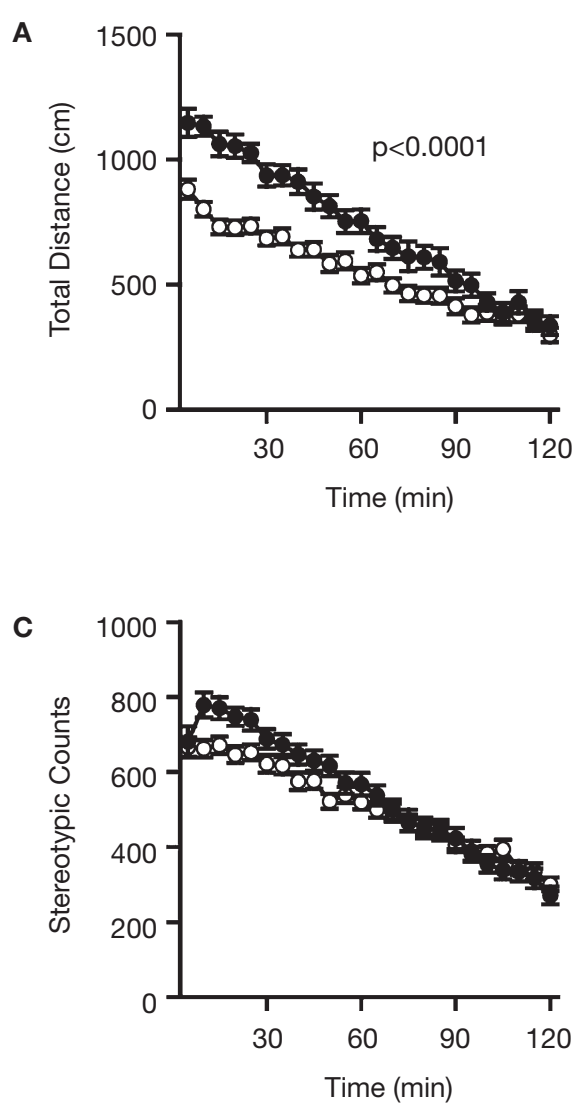
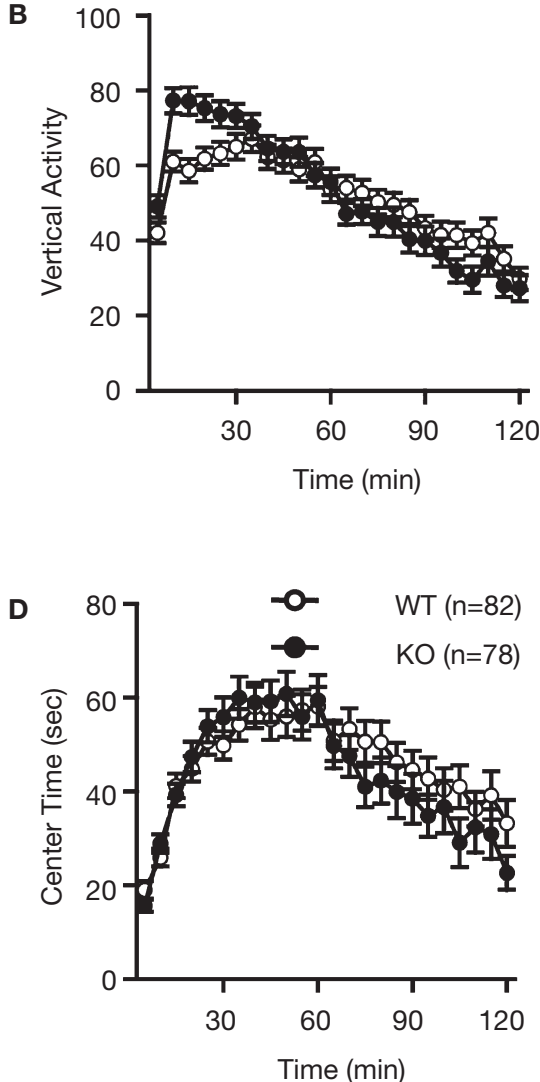

FIGURE 2 | Increased locomotor activity of RyR3-I- mice in the open field test. (A)Total locomotion distance was significantly increased in the KO mice. (B) Count of vertical activity. (C) Count of stereotypic behavior. (D) Time spent in the center of the compartment. WT and KO stand for RyR3+/+ and RyR3-/- mice, respectively. 
A

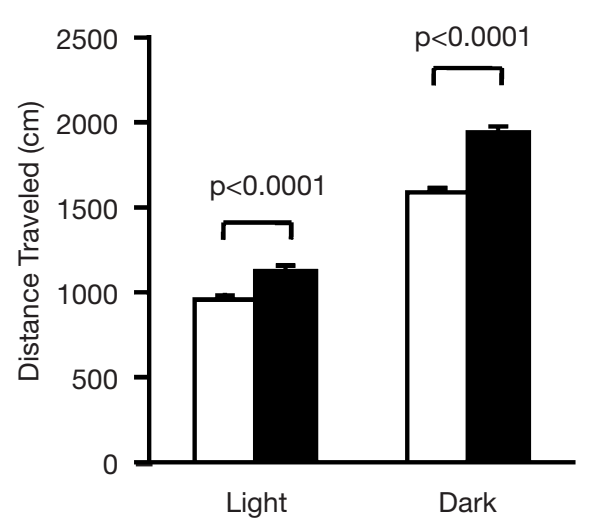

C

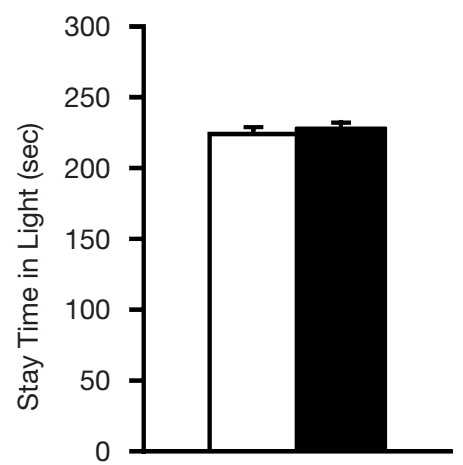

B

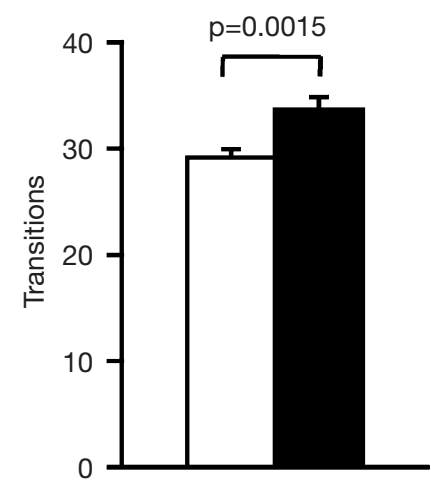

D

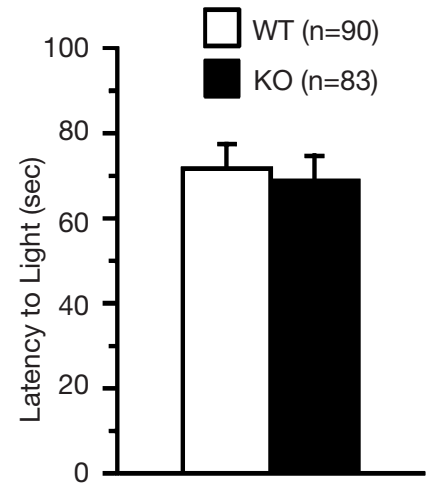

FIGURE 3 | Performance of RyR3-/- mice in the light/dark transition test. (A) Distance traveled in the light and dark sides. (B) Number of transitions between the light and dark sides. (C) Time the mice stayed in the light side. (D) Latency time before the first entry into the light side.

interaction test in a novel environment (Figure 6E; $F_{1,80}=39.033$, $p<0.0001)$. The number of transitions in the light-dark transition test (Figure 3B; $F_{1,171}=10.440, p=0.0015$ ) and the number of total entries in the elevated plus-maze test (Figure 4B; $F_{1,83}=12.707$, $p=0.0006)$ of the RyR3-/- mice were also significantly higher than those of controls. Vertical activity and stereotypic counts did not differ between the genotypes in the open field test (Figures 2B,C; $p=0.8906$, and 0.5725 , respectively). Even in the homecage, which is not a novel environment, the activity level of RyR3-/- mice was significantly greater relative to controls during the dark phase of the circadian cycle (Figure 7A; $F_{1,30}=10.046, p=0.0035$ ), indicating that the hyperactivity phenotype is caused by an increased general locomotor activity rather than an increased response to novelty.

Measures of anxiety-like behavior, such as the time spent in the central part of the open field (Figure 2D; $F_{1,159}=0.320, p=0.5725$ ), and in the light box of the light-dark transition test (Figure 3C; $F_{1,171}=0.274$, $p=0.6014$ ), did not differ significantly between the wild-type and the knockout mice although the time spent in the open arms in the elevated plus-maze test was greater in the knockout than that in the wild-type mice (Figure 4D; $F_{1,83}=4.737, p=0.0324$ ). First latency in the light-dark transition test (Figure 3D; $F_{1,171}=0.124, p=0.7257$ ) and entries into the open arms in the elevated plus-maze (Figure 4C; $F_{1,83}=3.472, p=0.0660$ ) were not significantly different between the genotypes. These observations suggest that RyR3 deficiency might decrease anxiety-like behavior, though the effect is subtle, if any.
We examined for depression-like behavior of RyR3-/- mice in the Porsolt forced swim test, which measures the time of immobility in a small pool that contains no means of escape. Mice will generally swim in the water but after a while will stop swimming and float on the surface of the water, appearing to have given up escaping. On the first day of the test, there was no significant difference in immobility between the mutant and wild-type mice (Figure 5A; $p=0.1483$ ), suggesting normal depression-like behavior in RyR3-/- mice. However, in the first 2 min of testing of trial 2 conducted 1 day after the first trial and of trial 3 conducted 7 days after the second trial, knockout mice showed less immobility relative to controls (Figure 5A; $F_{1,105}=11.979, p=0.0008$, and $F_{1,105}=6.122, p=0.0150$, respectively). Since the locomotor activity measured by distance traveled in the test did not differ between the knockout and the control on day 1 (Figure 5B; $F_{1,92}=1.81$, $p=0.1818)$, the results suggest that RyR3-/- mice did not learn and/or remember helplessness compared with wild-type mice.

\section{DECREASED SOCIAL INTERACTION IN RyR3-/- MICE}

During a social interaction test in a novel environment, the total duration of contacts and mean duration per contact of RyR3-/mice was significantly lower than that of wild-type mice (Figure 6A; $F_{1,80}=11.852, p=0.0009$, Figure 6D; $\left.F_{1,80}=6.263, p=0.0144\right)$ although the number of contacts, and total duration of active contacts, did not differ between genotypes (Figures 6B,C; $F_{1,80}=0.630$, 


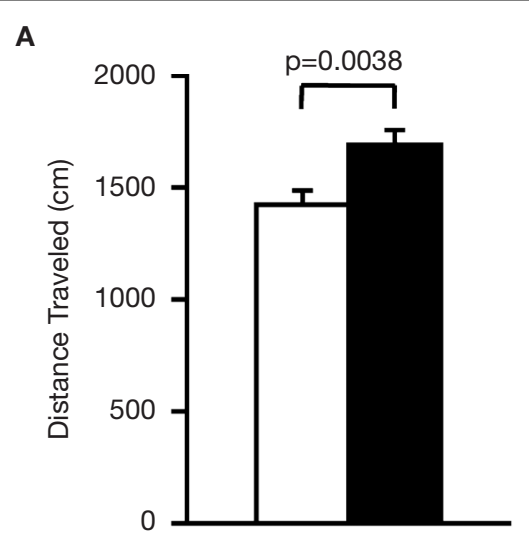

C

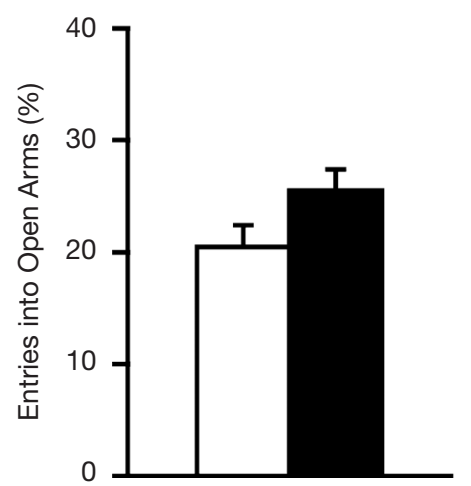

B

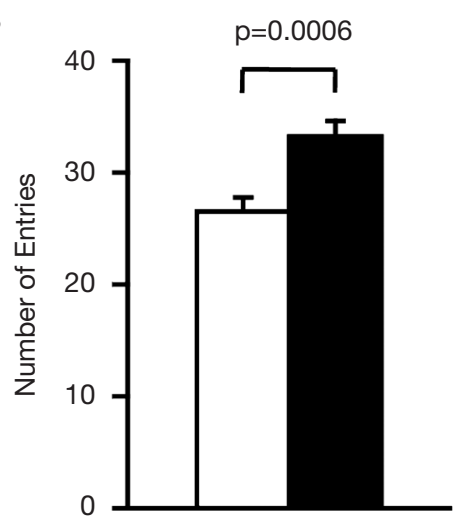

D

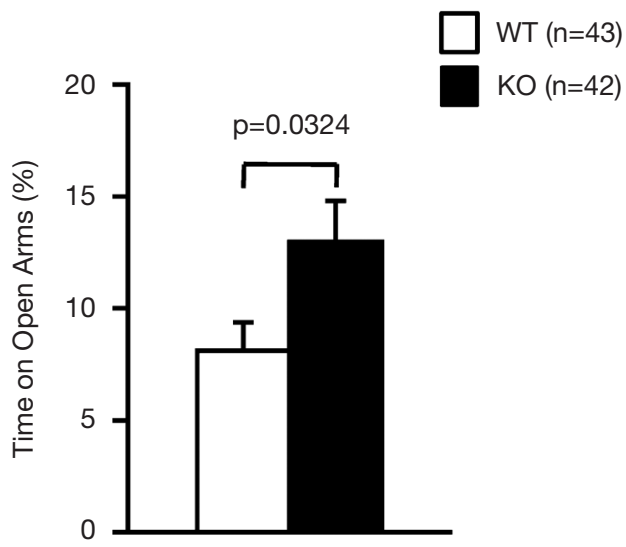

FIGURE 4 | Performance of RyR3-/- mice in the elevated plus-maze test. (A) Total distance traveled. (B) Number of entries into the center crossing between the open and closed arms. (C) Percent number of entries into the open arms. (D) Percent time spent on the open arms.

$p=0.4296$, and $F_{1,80}=1.434, p=0.2347$, respectively). It is possible that the decreased duration of contacts was due to a consequence of increased locomotion of the knockout mice since locomotor activity of the knockout mice was increased in this test (Figure 6E; $\left.F_{1,80}=39.033, p<0.0001\right)$. To confirm that the decrease in social interaction was not simply the result of hyperactivity in the RyR3 knockout mice, we monitored social interaction in the homecage over 5 days. During the light cycle when mice are usually sleeping and the activity level is similar between genotypes (Figure 7A; $\left.F_{1,30}=2.471, p=0.1264\right)$, the time that RyR3 knockout mice spent separated from each other was significantly greater than that of controls (Figure $7 \mathbf{B} ; F_{1,30}=5.482, p=0.0261$ ). No significant difference was observed in the mean number of particles between genotypes during the dark cycle (Figure $7 \mathbf{B} ; F_{1,30}=2.673, p=0.1125$ ). These results suggest that the decreased social interaction phenotype of the RyR3-/- mice is not simply a result of their hyper locomotor activity.

To further assess the social interaction behavior of RyR3 knockout mice, we performed the Crawley's three chamber sociability and social novelty preference test. In this test, we were not able to detect any significant differences between genotypes in exploratory behavior within either the first trial with one stranger mouse, nor the following trial with an additional stranger mouse (data deposited in the gene-brain-phenotyping database: https:// behav.hmro.med.kyoto-u.ac.jp/). In this test, a stranger mouse is tethered to a small wire cage while a stranger mouse can freely move in the social interaction test in a novel environment and in the homecage. This difference of the condition might lead to the seemingly different results in a social interaction behavior of the RyR3-/- mice.

\section{MILDLY IMPAIRED PREPULSE INHIBITION IN RyR3-/- MICE}

RyR3-/-mice were tested for sensorimotor gating deficits in the prepulse inhibition test. In normal mice, the startle reflex to a loud tone is attenuated by the presentation of a barely audible tone (prepulse) just before the loud tone. The amplitudes of the startle response were similar in wild-type and RyR3-/- mice before testing (Figure 8A; $\left.F_{1,175}=1.592, p=0.2088\right)$. Although the overall genotype effect of percentage prepulse inhibition was not significant (Figure 8B; $\left.F_{1,175}=2.581, p=0.1099\right)$, the prepulse inhibition at $78 \mathrm{~dB}$ prepulse to $110 \mathrm{~dB}$ startle in the knockouts was significantly lower than that in the wild-type mice (Figure 8B; $F_{1,175}=11.906, p=0.0007$ ), indicating a mildly impaired prepulse inhibition in RyR3 knockout mice.

\section{MILDLY IMPAIRED LATENT INHIBITION IN RyR3-I- MICE}

The RyR3-/- mice were examined in the latent inhibition test (Figures 9A-F). Latent inhibition refers to the phenomenon by which preexposure to stimuli (such as tones) retards subsequent conditioning to 

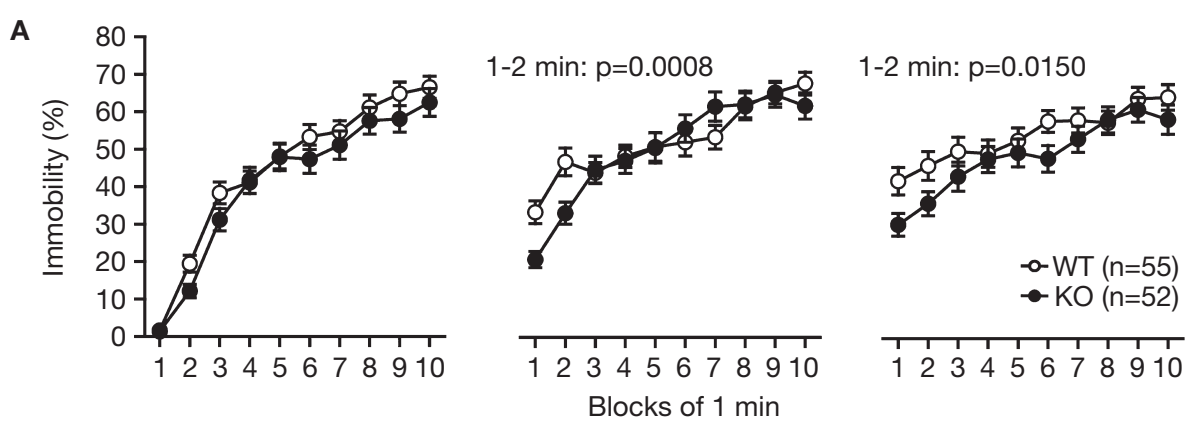

- WT $(n=55)$

$\bullet \mathrm{KO}(\mathrm{n}=52)$

$\begin{array}{llllllllll}12 & 3 & 4 & 5 & 6 & 7 & 8 & 9 & 10\end{array}$

B
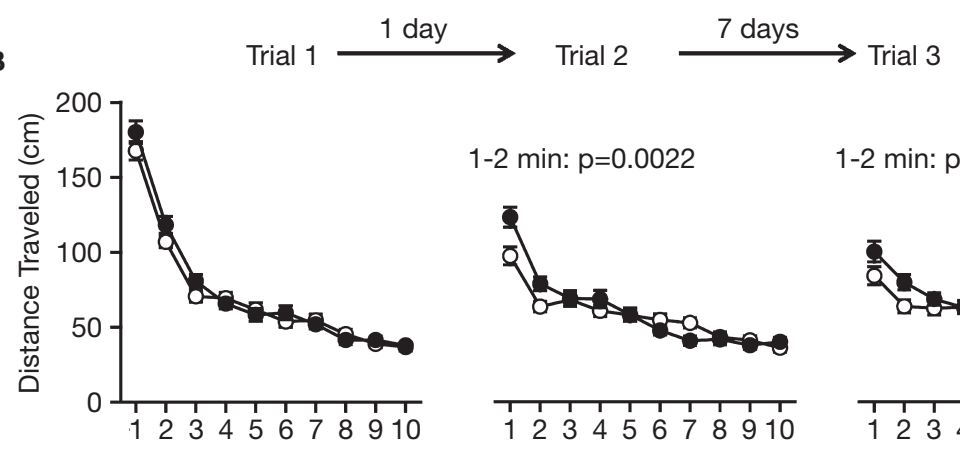

1-2 min: $p=0.0022$

$1-2 \min : p=0.0243$
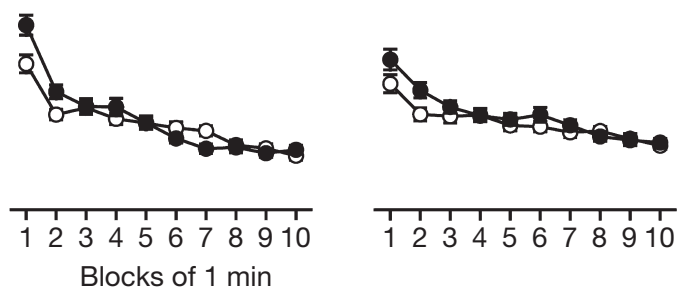

FIGURE 5 | Performance of RyR3-/- mice in the Porsolt forced swim test. (A) Immobility time (\%) in each block for trial 1, trial 2, and trial 3. (B) Total distance traveled in each block for trial 1, trial 2, and trial 3.

A

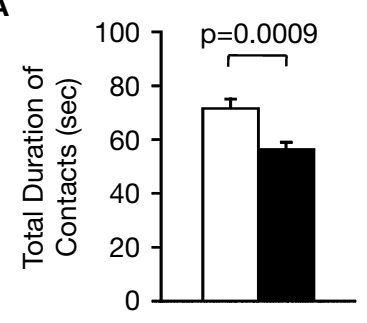

C

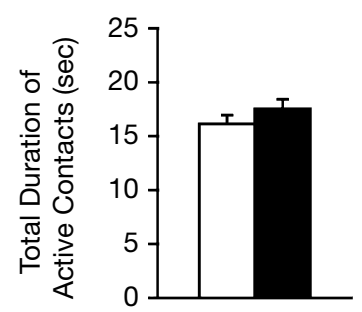

B

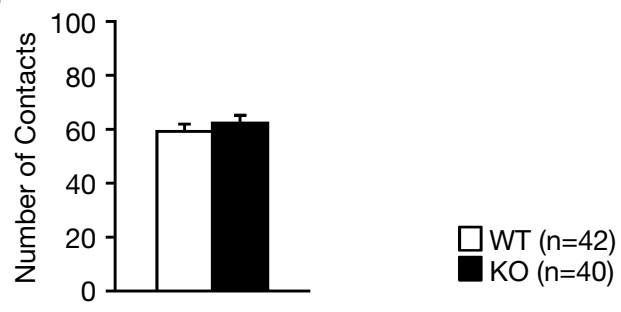

D

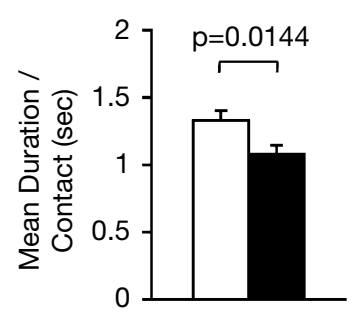

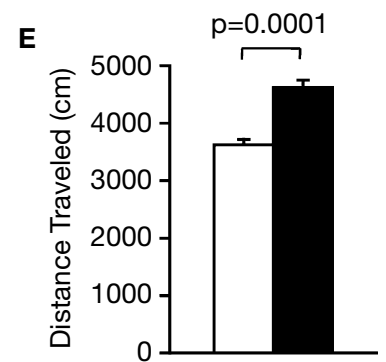

FIGURE 6 | Decreased social interaction in a novel environment. (A) Decreased total duration of contacts in the KO mice. (B) Number of contacts. (C) Total duration of active contacts. (D) Mean duration per contact. (E) Total distance traveled.

the same stimulus. The reduced conditioning is believed to result from attentional filtering so that decreased attention is given to a familiar stimulus that the animal has learned to ignore during pre-exposure. Wild-type mice that were pre-exposed $(\mathrm{P})$ to the tone showed less freezing to the tone on the test day compared to non pre-exposed (NP) animals (Figure 9C; $F_{1,55}=6.042, p=0.0171$ ), indicating significant latent inhibition in control mice. The freezing percent of pre-exposed RyR3-/- mice was lower than that of non-exposed RyR3-/- mice though it was not significant (Figure 9F; $F_{1,53}=3.877, p=0.0542$ ), suggesting a mild deficit of RyR3 mutant mice in latent inhibition. 

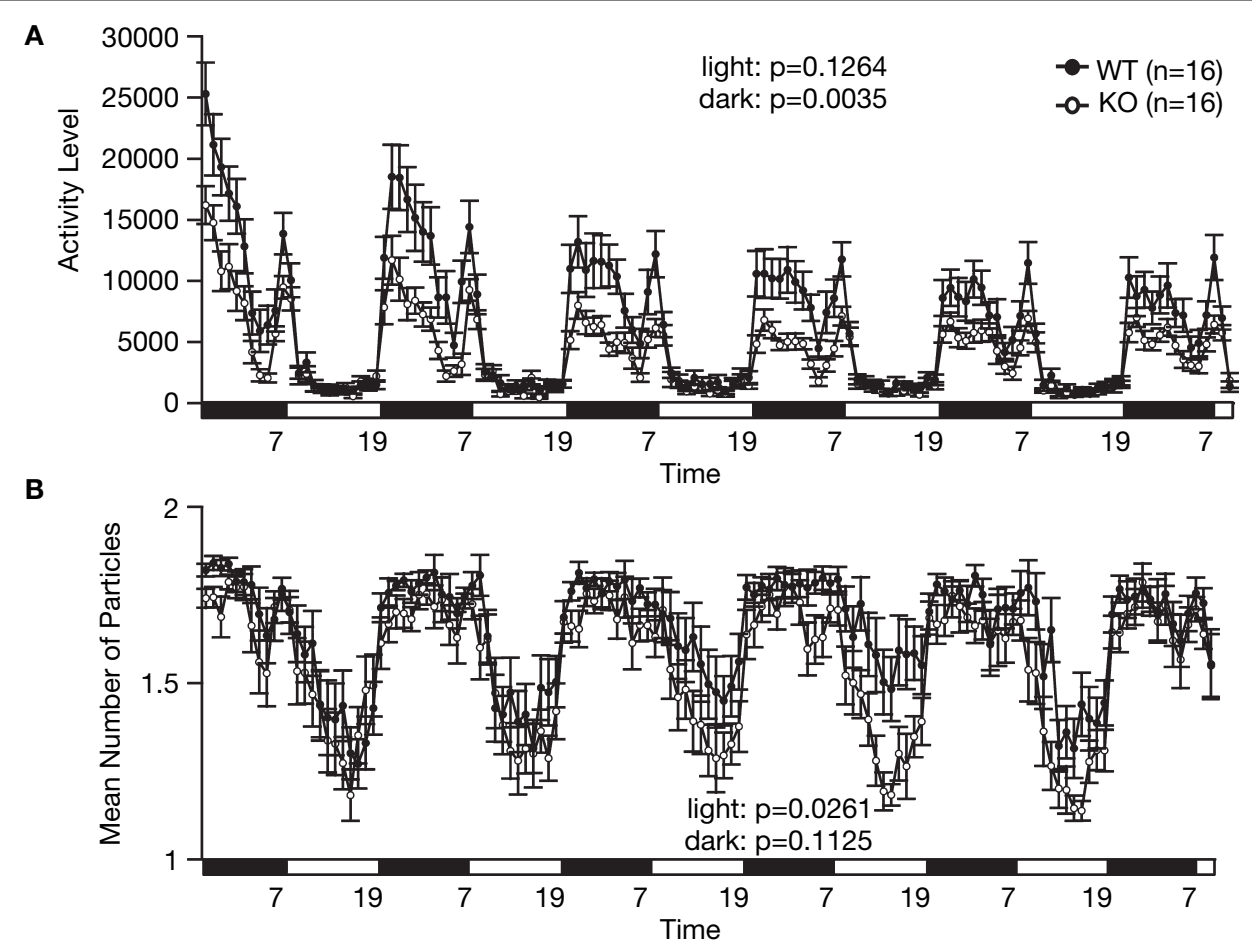

FIGURE 7 | Decreased social interaction in the home cage. (A) An index of activity level. (B) Number of particles in the home cage. Social interaction is expressed as the number of particles in the image of the cage found by automatic detection. When the animals are separated, the particle number is 2 .
When they are together, the number is 1. Each dot indicates the average particles per hour. During the light cycle (7:00 to 19:00), WT mice stayed in contact with their familiar cage mate, whereas the KO mice tended to stay separated from each other.
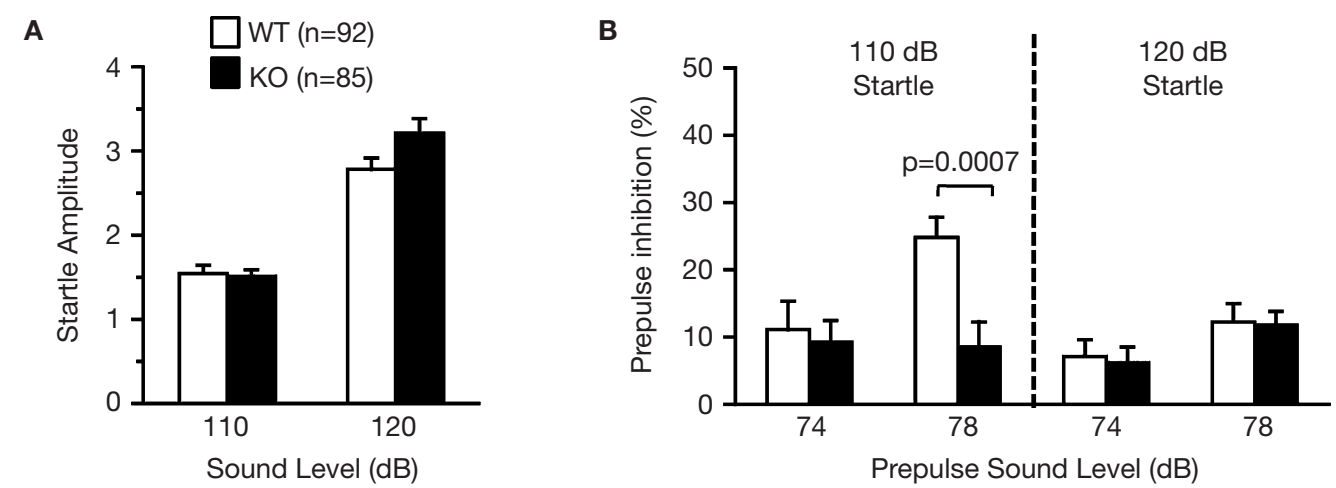

FIGURE 8 | Mildly impaired prepulse inhibition of RyR3-/- mice. (A) Acoustic startle response was not significantly different between genotypes. (B) Prepulse inhibition at $78 \mathrm{~dB}$ prepulse to $110 \mathrm{~dB}$ startle in the knockouts was significantly lower than that in the wild-type mice.

\section{NORMAL WORKING AND REFERENCE MEMORIES IN 8-ARM RADIAL MAZE IN RyR3-I-}

To further examine whether the loss of RyR3 was associated with cognitive deficits, we analyzed RyR3-/- mice in working memory and reference memory tasks. To assess working memory, we used a spatial working memory version of the eight-arm radial maze task, known to be dependent on the hippocampus (Becker et al., 1980). In this task, animals must learn and remember the position of baited arms between trials while rapidly establishing memory of previously visited arms within a trial. Over training, mutant mice improved their performance equally well as wild-type mice as indicated by a progressive reduction in the number of revisiting errors (Figure 10A; $\left.F_{1,45}=1.025, p=0.3168\right)$. After training, there was no significant difference in the number of revisiting errors during trials with a delay of 30,120 , or $300 \mathrm{~s}$ (Figure 10B; $F_{1,45}=1.254, p=0.2686, F_{1,45}=0.013$, $p=0.9094, F_{1,45}=0.210, p=0.6488$, respectively). Furthermore, the number of different arms chosen during the first eight choices, which is considered a measure of working memory that is relatively independent of locomotor activity levels and the total number of choices, was not significantly affected by the deficit of RyR3 during training 


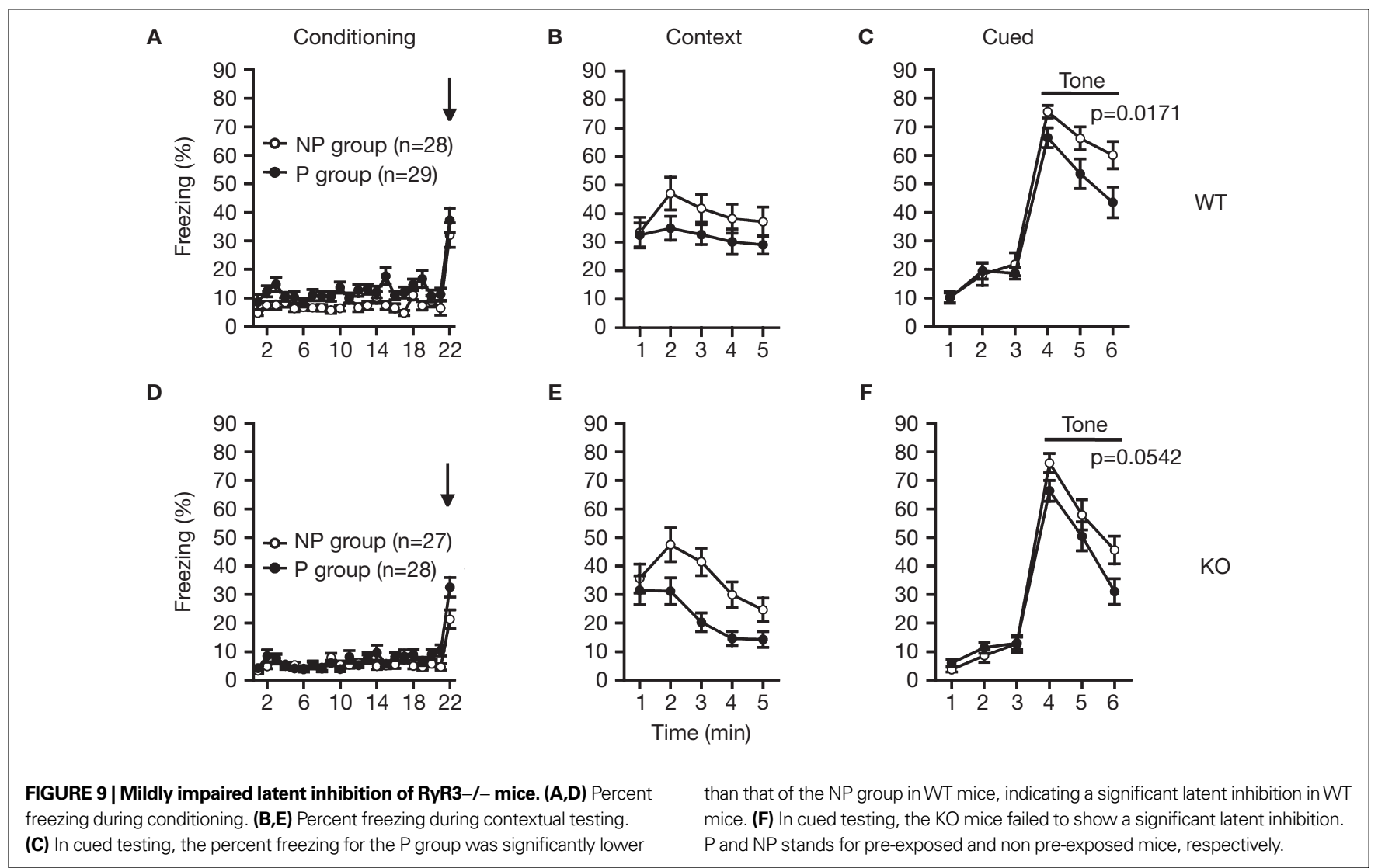

A

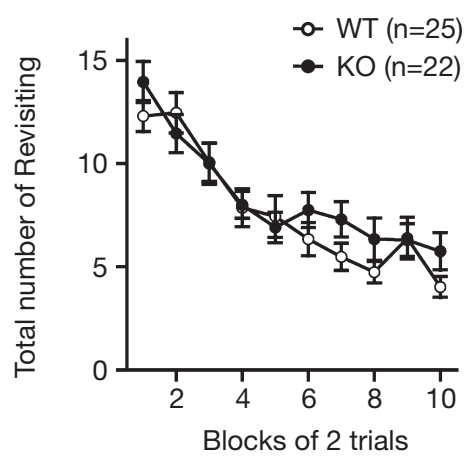

C

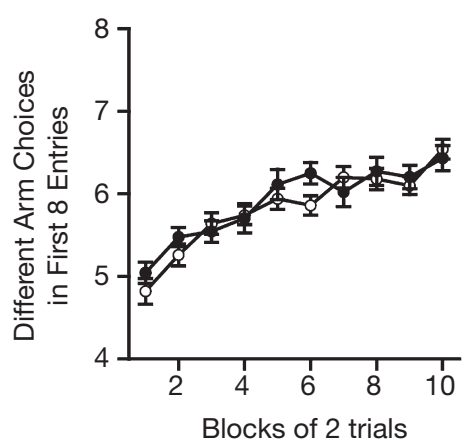

B

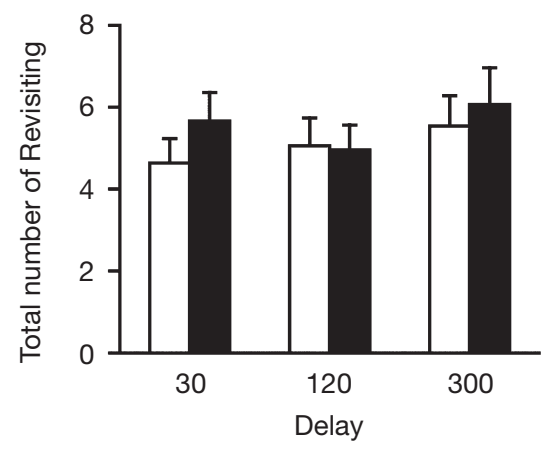

D

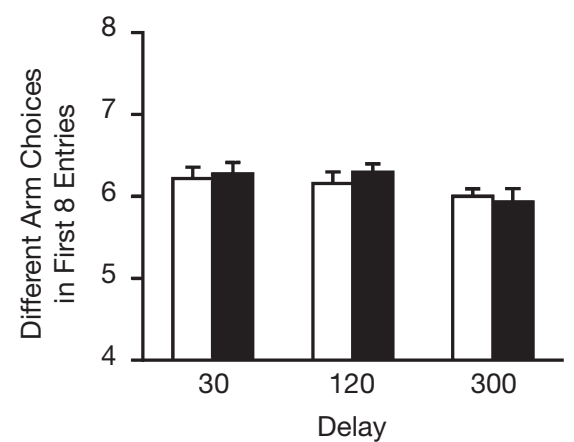

FIGURE 10 | Intact spatial working memory ability in the eight-arm radial maze task. (A,B) Number of revisiting errors. (C,D) Number of different arms chosen within the first eight choices. 


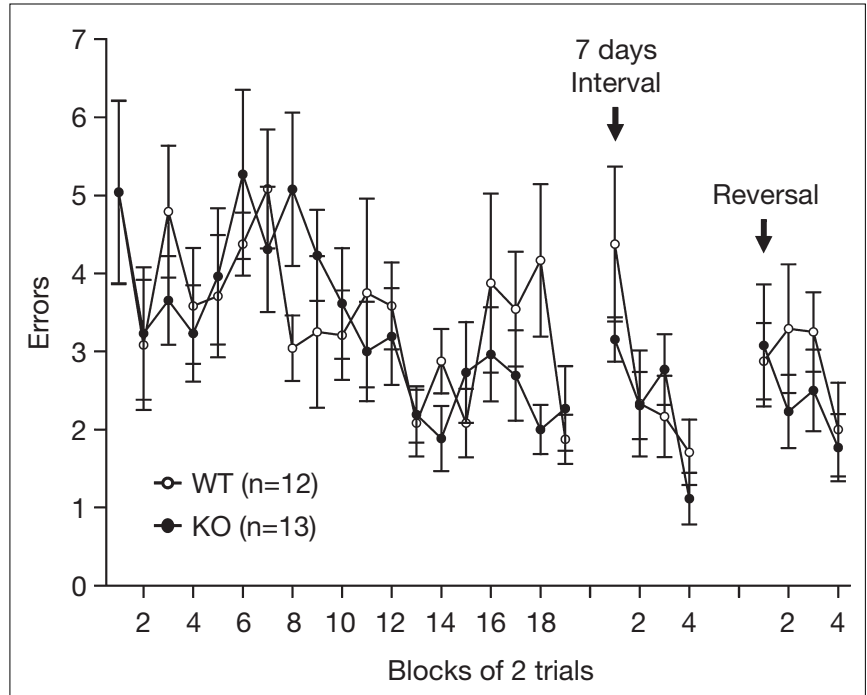

FIGURE 11 | Intact spatial reference memory ability in the eight-arm

radial maze task. Number of errors made before finding the fixed baited arm during spatial learning, memory retention, and relearning.

(Figure 10C; $F_{1,45}=0.723, p=0.3995$ ) and trials with $30,120,300 \mathrm{~s}$ of delay (Figure 10D; $F_{1,45}=0.72, p=0.7900, F_{1,45}=0.597, p=0.4438$, $F_{1,45}=0.143, p=0.7073$, respectively). These results demonstrate that RyR3-/- mice exhibit normal ability in working memory.

The spatial reference memory of RyR3-I- mice was also examined using the eight-arm radial maze. Both wild-type and mutant mice showed similar performance in learning the fixed position of the baited arm (Figure 11). Thus the spatial reference memory does not seem to be affected by the lack of RyR3. To evaluate memory retention, mice were retested in the same radial maze after a period of 7-day in their home cage. During retention testing, wild-type and knockout mice did not differ significantly in the number of errors made before finding the target arm (Figure 11). We further tested the ability of relearning by reversal training, in which the radial maze is rebaited in the opposite arm. There was no significant difference between the wild-type and knockout mice in the number of errors (Figure 11).

\section{DISCUSSION}

The lack of RyR3 did not lead to significant abnormalities in overall health, appearance, and basic sensorimotor functions. However, RyR3-/- mice demonstrated some significant abnormal behaviors. Hyperlocomotor activity is a characteristic feature of RyR3-/- mice as previously described (Takeshima et al., 1996). The increase in locomotion of RyR3-/- mice in various tests is primarily caused by their hyperactivity rather than an altered anxiety-like behavior and/ or curiosity to a novel environment because we did not see robust changes of anxiety-like behavior in RyR3-/- mice and we observed an increased basal locomotor activity in their familiar home cage. Regarding the anxiety-like behavior of RyR3-/- mice, a previous study showed an increased time spent on the open arms in the elevated plus-maze test, suggesting reduced fear or anxiety (Kouzu et al., 2000). Our RyR3-/- mice also showed a slight increase in the time spent on the open arms in the elevated plus-maze test compared to the wild-type mice. However, it is unlikely that RyR3 plays a major role in controlling anxiety-like behaviors in mice since the knockout mice failed to show significant differences in multiple tests including the open field test, and light-dark transition test.

One of the most striking abnormal behaviors of RyR3-/- mice is decreased social interaction. The decrease of total duration of contacts and mean duration per contact in a novel environment observed in RyR3-/- mice could be a result of hyperlocomotion rather than decreased social interaction. However, our meta analysis of C57BL/6J mice revealed no significant correlations between the total duration of contacts and the total distance traveled $(r=-0.13, n=935 \mathrm{C} 57 \mathrm{Bl} / 6 \mathrm{~J}$ mice; $r=0.06, n=1723$ mice including various mutant mice; Takao and Miyakawa, unpublished data), indicating that the hyperactive phenotype does not generally affect the total duration of contacts in the social interaction test in a novel environment. Moreover, the social interaction test in the home cage revealed that RyR3-/- mice tended to sleep alone. These results suggest that RyR3 deficiency affects social behavior. In humans, decreased social interaction is a key symptom of several psychiatric diseases such as schizophrenia and autism spectrum conditions (American Psychiatric Association, 2000). The human RyR3 gene has been mapped to chromosome 15q14-q15 (Sorrentino et al., 1993) and the chromosomal region has been suggested to harbor schizophrenia and autism susceptibility genes (Abrahams and Geschwind, 2008; Leonard and Freedman, 2006; Liu et al., 2001; Moon et al., 2006; Williams et al., 1999). These facts suggest that the RyR3 gene is a candidate for susceptibility to these disorders, although a significant association was not found in the Japanese population (Tochigi et al., 2008; Yamada et al., 2007). Previously, we found increased social interactions in cyclic nucleotide phosphodiesterase 10A2 (PDE10A2) knockout mice without any other major behavioral deficits (Sano et al., 2008). This suggests that a PDE10A inhibitor could be effective for the treatment of negative symptoms of schizophrenia, including social withdrawal. Similarly, RyR3 might be a candidate target for these psychiatric disorders with negative symptoms. Further investigation would be required to address the possibility.

The mechanisms underlying decreased social behavior of RyR3 knockout mice are still unknown. Oxytocin could be one of the key molecules involved in the deficit of social interaction in RyR3 knockout mice. Oxytocin is a neuropeptide that is known to play a critical role in the regulation of social recognition (Choleris et al., 2006; Ferguson et al., 2000). Recently, an involvement of $\mathrm{Ca}^{2+}$ release from RyRs in oxytocin secretion was suggested (Jin et al., 2007). Thus, there is a possibility that the lack of RyR3 might cause a deficit in oxytocin system, and consequently abnormal social behaviors.

Previous studies using the Morris water maze demonstrated that RyR3-/- mice spent the same time or slightly more time in the target quadrant compared with the wild-type mice after training (Balschun et al., 1999; Futatsugi et al., 1999), suggesting a normal or slightly better spatial reference memory in RyR3-/- mice. Interestingly, the performance was impaired during reversal learning of a new platform position (Balschun et al., 1999), implying a reduced flexibility or increased perseveration of RyR3-/- mice. In contrast, we did not detect significant differences between the knockout and wild-type mice during either learning or reversal learning in the spatial reference memory version of the eight-arm radial maze. The Morris 
water maze is the most frequently used paradigm to assess a spatial reference memory that is dependent on the hippocampus. However, it is a highly stressful task. Thus it is possible that RyR3-/- mice may have an abnormal sensitivity to stress and show stronger perseveration under the stressful conditions. Consistently, our result suggests that RyR3-/- mice show poor learning and/or memory for depression-like behavior in the Porsolt forced swim test. Alternatively, the discrepancy of the reversal learning performance may be due to a difference of genetic background.

Previous reports demonstrated a decreased alteration performance of RyR3-/- mice in the Y-maze test (Kouzu et al., 2000). The authors concluded that RyR3-/- mice exhibited defects of spatial working memory. However, the Y-maze test may not be a suitable task to evaluate working memory since it is assessed by a simple spontaneous alternation behavior that is often affected by various factors such as perseveration, restricted interests, motivation, and serial search strategy. We examined the working memory performance of RyR3-/- mice using an eight-arm radial maze task. In this task, animals must rapidly establish and maintain a memory of the arms visited based on single within-trial exposures and must also suppress interference by the memory obtained during previous trials. This task is more difficult than the Y-maze task and is likely to evaluate spatial working memory more reliably. Since RyR3-/-mice exhibited normal performance in our eight-arm radial maze task, we conclude that the RyR3 deficiency does not cause an impaired ability of working memory in mice.

In many areas of the brain, more than one RyR isoform is redundantly expressed. Nevertheless, deficiency of one isoform,

\section{REFERENCES}

Abrahams, B. S., and Geschwind, D. H. (2008). Advances in autism genetics: on the threshold of a new neurobiology. Nat. Rev. Genet. 9, 341-355.

American Psychiatric Association (2000). Diagnostic and Statistical Manual of Mental Disorders (DSMIV-TR), 4th Edn (Text Revision). Washington, DC, American Psychiatric Association.

Arguello, P. A., and Gogos, J. A. (2006). Modeling madness in mice: one piece at a time. Neuron 52, 179-196.

Arron, J. R., Winslow, M. M., Polleri, A., Chang, C. P., Wu, H., Gao, X., Neilson, J. R., Chen, L., Heit, J. J., Kim, S. K., et al. (2006). NFAT dysregulation by increased dosage of DSCR1 and DYRK1A on chromosome 21. Nature 441, 595-600.

Balschun, D., Wolfer, D. P., Bertocchini, F., Barone, V., Conti, A., Zuschratter, W., Missiaen, L., Lipp, H. P., Frey, J. U., and Sorrentino, V. (1999). Deletion of the ryanodine receptor type 3 (RyR3) impairs forms of synaptic plasticity and spatial learning. EMBO J. 18, 5264-5273.

Bardo, S., Cavazzini, M. G., and Emptage, N. (2006). The role of the endoplasmic reticulum $\mathrm{Ca} 2+$ store

RyR3, leads to various behavioral abnormalities as discussed above. There are reports showing a functional difference of distinct RyR isoforms. For example, short-term synaptic plasticity induced by short tetanic stimulation is unchanged in RyR3-/- mice (Shimuta et al., 2001), suggesting that the activation of RyR3 requires stronger synaptic activity. Consistent with this idea, RyR3 has a lower sensitivity to $\mathrm{Ca}^{2+}$ compared with other isoforms (Ikemoto et al., 1997; Imagawa et al., 1992; Takeshima et al., 1995, 1996). The distinct character of RyR3 in synaptic plasticity might be important for specific neuronal functions involved in behaviors including hyperactivity, social interaction, prepulse inhibition, and latent inhibition. It also suggests a critical role of the precise spatio-temporal regulation of internal $\mathrm{Ca}^{2+}$ concentrations in neurons and thus a lack of one molecule involved in the signaling pathway results in abnormal behaviors in mice.

\section{ACKNOWLEDGEMENTS}

This work was supported by KAKENHI (Grant-in-Aid for Scientific Research) on Priority Areas 'Systems Genomics' (20016013), on Priority Areas 'Pathomechanisms of Brain Disorders' (20023017), Young Scientists A (16680015), Exploratory Research (19653081), and Integrative Brain Research (IBR-shien) from the Ministry of Education, Culture, Sports, Science and Technology (MEXT) of Japan, Promotion of Fundamental Studies in Health Sciences of the National Institute of Biomedical Innovation (NIBIO), Neuroinformatics Japan Center (NIJC), and by grants from CREST \& BIRD of Japan Science and Technology Agency (JST).

alpha, beta and oxytocin in social discrimination: a detailed behavioral analysis with knockout female mice. Genes Brain Behav. 5, 528-539.

Ferguson, J. N., Young, L. J., Hearn, E. F., Matzuk, M. M., Insel, T. R., and Winslow, J. T. (2000). Social amnesia in mice lacking the oxytocin gene. Nat. Genet. 25, 284-288.

Ferris, C. D., and Snyder, S. H. (1992). Inositol 1,4,5-trisphosphate-activated calcium channels. Annu. Rev. Physiol. 54, 469-488.

Futatsugi, A., Kato, K., Ogura, H., Li, S. T., Nagata, E., Kuwajima, G., Tanaka, K., Itohara, S., and Mikoshiba, K. (1999). Facilitation of NMDAR-independent LTP and spatial learning in mutant mice lacking ryanodine receptor type 3. Neuron 24, 701-713.

Gainetdinov, R. R., Mohn, A. R., and Caron, M. G. (2001). Genetic animal models: focus on schizophrenia. Trends Neurosci. 24, 527-533.

Gerber, D. J., Hall, D., Miyakawa, T., Demars, S., Gogos, J. A., Karayiorgou, M., and Tonegawa, S. (2003). Evidence for association of schizophrenia with genetic variation in the $8 \mathrm{p} 21.3$ gene, $\mathrm{PPP} 3 \mathrm{CC}$, encoding the calcineurin gamma subunit. Proc. Natl. Acad. Sci. USA 100, 8993-8998.
Giannini, G., Conti, A., Mammarella, S., Scrobogna, M., and Sorrentino, V. (1995). The ryanodine receptor/calcium channel genes are widely and differentially expressed in murine brain and peripheral tissues. J. Cell Biol. 128, 893-904.

Horiuchi, Y., Ishiguro, H., Koga, M., Inada, T., Iwata, N., Ozaki, N., Ujike, H., Muratake, T., Someya, T., and Arinami, T. (2007). Support for association of the PPP3CC gene with schizophrenia. Mol. Psychiatry 12, 891-893.

Ikemoto, T., Komazaki, S., Takeshima, H., Nishi, M., Noda, T., Iino, M., and Endo, M. (1997). Functional and morphological features of skeletal muscle from mutant mice lacking both type 1 and type 3 ryanodine receptors. J. Physiol. 501(Pt 2), 305-312.

Imagawa, T., Nakai, J., Takeshima, H., Nakasaki,Y., and Shigekawa, M. (1992). Expression of $\mathrm{Ca}(2+)$-induced $\mathrm{Ca} 2+$ release channel activity from cardiac ryanodine receptor cDNA in Chinese hamster ovary cells. J. Biochem. 112, 508-513.

Choleris, E., Ogawa, S., Kavaliers, M., Gustafsson, J. A., Korach, K. S., Muglia, L. J., and Pfaff, D. W. (2006). Involvement of estrogen receptor
Jin, D., Liu, H. X., Hirai, H., Torashima, T., Nagai, T., Lopatina, O., Shnayder, N. A., Yamada, K., 
Noda, M., Seike, T., et al. (2007). CD38 is critical for social behaviour by regulating oxytocin secretion. Nature 446, 41-45.

Komada, M., Takao, K., and Miyakawa, T. (2008). Elevated plus maze for mice. J. Vis. Exp. 1088. doi: 10.3791/1088.

Kouzu, Y., Moriya, T., Takeshima, H., Yoshioka, T., and Shibata, S. (2000). Mutant mice lacking ryanodine receptor type 3 exhibit deficits of contextual fear conditioning and activation of calcium/calmodulindependent protein kinase II in the hippocampus. Brain Res. Mol. Brain Res. 76, 142-150.

Kuwajima, G., Futatsugi, A., Niinobe, M., Nakanishi, S., and Mikoshiba, K. (1992). Two types of ryanodine receptors in mouse brain: skeletal muscle type exclusively in Purkinje cells and cardiac muscle type in various neurons. Neuron 9, 1133-1142.

Leonard, S., and Freedman, R. (2006). Genetics of chromosome 15q13-q14 in schizophrenia. Biol. Psychiatry 60, 115-122.

Liu, C. M., Hwu, H. G., Lin, M. W., OuYang, W. C., Lee, S. F., Fann, C. S., Wong, S. H., and Hsieh, S. H. (2001). Suggestive evidence for linkage of schizophrenia to markers at chromosome 15q13-14 in Taiwanese families. Am. J. Med. Genet. 105, 658-661.

Liu, Y. L., Fann, C. S., Liu, C. M., Chang, C. C., Yang, W. C., Hung, S. I., Yu, S. L., Hwang, T. J., Hsieh, M. H., Liu, C. C., et al. (2007). More evidence supports the association of PPP3CC with schizophrenia. Mol. Psychiatry 12, 966-974.

Mathieu, F., Miot, S., Etain, B., E1 Khoury, M. A., Chevalier, F., Bellivier, F., Leboyer, M., Giros, B., and Tzavara, E. T. (2008). Association between the PPP3CC gene, coding for the calcineurin gamma catalytic subunit, and bipolar disorder. Behav. Brain Funct. 4, 2.

McPherson, P. S., and Campbell, K. P. (1993). The ryanodine receptor/Ca2+ release channel. J. Biol. Chem. 268, 13765-13768.

Mikoshiba, K. (1993). Inositol 1,4,5trisphosphate receptor. Trends Pharmacol. Sci. 14, 86-89.

Miyakawa, T., Leiter, L. M., Gerber, D. J. Gainetdinov, R. R., Sotnikova, T. D., Zeng, H., Caron, M. G., and Tonegawa, S. (2003). Conditional calcineurin knockout mice exhibit multiple abnormal behaviors related to schizophrenia. Proc. Natl. Acad. Sci. USA 100, 8987-8992.

Moon, H. J., Yim, S. V., Lee, W. K. Jeon, Y. W., Kim, Y. H., Ko, Y. J., Lee, K. S., Lee, K. H., Han, S. I., and Rha, H. K. (2006). Identification of DNA copy-number aberrations by array-comparative genomic hybridization in patients with schizophrenia. Biochem. Biophys. Res. Commun. 344, 531-539.

Mori, F., Fukaya, M., Abe, H., Wakabayashi, K., and Watanabe, $\mathrm{M}$. (2000). Developmental changes in expression of the three ryanodine receptor mRNAs in the mouse brain. Neurosci. Lett. 285, 57-60.

Powell, C. M., and Miyakawa, T. (2006). Schizophrenia-relevant behavioral testing in rodent models: a uniquely human disorder? Biol. Psychiatry 59, 1198-1207.

Rose, C. R., and Konnerth, A. (2001). Stores not just for storage: intracellular calcium release and synaptic plasticity. Neuron 31, 519-522.

Sano, H., Nagai, Y., Miyakawa, T., Shigemoto, R., and Yokoi, M. (2008) Increased social interaction in mice deficient of the striatal medium spiny neuron-specific phosphodiesterase 10A2. J. Neurochem. 105, 546-556.

Shi, J., Gershon, E. S., and Liu, C. (2008). Genetic associations with schizophrenia: meta-analyses of 12 candidate genes. Schizophr. Res. 104, 96-107.

Shimuta, M., Yoshikawa, M., Fukaya, M., Watanabe, M., Takeshima, H., and Manabe, T. (2001). Postsynaptic modulation of AMPA receptor-mediated synaptic responses and LTP by the type 3 ryanodine receptor. Mol. Cell. Neurosci. 17, 921-930.

Snyder, S. H., Lai, M. M., and Burnett, P.E. (1998). Immunophilins in the nervous system. Neuron 21, 283-294.

Sorrentino, V., Giannini, G., Malzac, P., and Mattei, M.G. (1993). Localization of a novel ryanodine receptor gene (RyR3) to human chromosome 15q14-q15 by in situ hybridization. Genomics 18, 163-165.

Sorrentino, V., and Volpe, P. (1993). Ryanodine receptors: how many, where and why? Trends Pharmacol. Sci. 14, 98-103.

Takao, K., and Miyakawa, T. (2006a). Investigating gene-to-behavior pathways in psychiatric disorders: the use of a comprehensive behavioral test battery on genetically engineered mice. Ann. NY Acad. Sci. 1086, 144-159.

Takao, K., and Miyakawa, T. (2006b). Light/dark transition test for mice. J. Vis. Exp. 104. doi: 10.3791/1088.

Takao, K., Yamasaki, N., and Miyakawa, T. (2007). Impact of brain-behavior phenotyping of genetically-engineered mice on research of neuropsychiatric disorders. Neurosci. Res. 58 124-132.

Takeshima, H., Iino, M., Takekura, H., Nishi, M., Kuno, J., Minowa, O. Takano, H., and Noda, T. (1994). Excitation-contraction uncoupling and muscular degeneration in mice lacking functional skeletal muscle ryanodine-receptor gene. Nature 369 556-559.

Takeshima, H., Ikemoto, T., Nishi, M., Nishiyama, N., Shimuta, M. Sugitani, Y., Kuno, J., Saito, I., Saito, H. Endo, M., et al. (1996). Generation and characterization of mutant mice lacking ryanodine receptor type 3. J. Biol Chem. 271, 19649-19652.

Takeshima, H., Komazaki, S., Hirose, K. Nishi, M., Noda, T., and Iino, M. (1998). Embryonic lethality and abnormal cardiac myocytes in mice lacking ryanodine receptor type 2 . EMBO J. 17, 3309-3316.

Takeshima,H., Yamazawa, T., Ikemoto, T., Takekura, H., Nishi, M., Noda, T. and lino, M. (1995). Ca(2+)-induced $\mathrm{Ca} 2+$ release in myocytes from dyspedic mice lacking the type-1 ryanodine receptor. EMBO J. 14 2999-3006.

Tochigi, M., Kato, C., Ohashi, J., Koishi, S. Kawakubo, Y., Yamamoto, K., Matsumoto, H., Hashimoto, O. Kim, S. Y., Watanabe, K., et al. (2008) No association between the ryanodine receptor 3 gene and autism in a Japanese population. Psychiatry Clin. Neurosci. 62, 341-344.

Wan, K., Moriya, T., Akiyama, M. Takeshima, H., and Shibata, S. (1999) Involvement of ryanodine receptor type 3 in dopamine release from the striatum: evidence from mutant mice lacking this receptor. Biochem. Biophys. Res. Commun. 266, 588-592.

Williams, N. M., Rees, M. I., Holmans, P. Norton, N., Cardno, A. G., Jones, L. A., Murphy, K. C., Sanders, R. D., McCarthy, G., Gray, M. Y., et al. (1999). A two-stage genome scan for schizophrenia susceptibility genes in
196 affected sibling pairs. Hum. Mol Genet. 8, 1729-1739.

Yamada, K., Gerber, D. J., Iwayama, Y., Ohnishi, T., Ohba, H., Toyota, T., Aruga, J., Minabe, Y., Tonegawa, S., and Yoshikawa, T. (2007). Genetic analysis of the calcineurin pathway identifies members of the EGR gene family, specifically EGR3, as potential susceptibility candidates in schizophrenia. Proc. Natl. Acad. Sci. USA 104, 2815-2820.

Yamasaki, N., Maekawa, M., Kobayashi, K., Kajii, Y., Maeda, J., Soma, M., Takao, K., Tanda, K. Ohira, K., Toyama, K., et al. (2008). Alpha-CaMKII deficiency causes immature dentate gyrus, a novel candidate endophenotype of psychiatric disorders. Mol. Brain 1, 6 .

Zalk, R., Lehnart, S. E., and Marks, A. R. (2007). Modulation of the ryanodine receptor and intracellular calcium. Annu. Rev. Biochem. 76, 367-385.

Zeng, H., Chattarji, S., Barbarosie, M., Rondi-Reig, L., Philpot, B. D., Miyakawa, T., Bear, M. F., and Tonegawa, S. (2001). Forebrainspecific calcineurin knockout selectively impairs bidirectional synaptic plasticity and working/episodic-like memory. Cell 107, 617-629.

Conflict of Interest Statement: The authors declare that the research was conducted in the absence of any commercial or financial relationships that could be construed as a potential conflict of interest.

Received: 21 January 2009; paper pending published: 17 February 2009; accepted: 06 April 2009; published online: 07 May 2009. Citation: Matsuo N, Tanda K, Nakanishi $K$, Yamasaki N, Toyama K, Takao K, Takeshima $H$ and Miyakawa $T$ (2009) Comprehensive behavioral phenotyping of ryanodine receptor type 3 ( $R y R 3)$ knockout mice: decreased social contact duration in two social interaction tests. Front. Behav. Neurosci. (2009) 3:3. doi: 10.3389/neuro.08.003.2009

Copyright (C) 2009 Matsuo, Tanda, Nakanishi, Yamasaki, Toyama, Takao, Takeshima and Miyakawa. This is an open-access article subject to an exclusive license agreement between the authors and the Frontiers Research Foundation, which permits unrestricted use, distribution, and reproduction in any medium, provided the original authors and source are credited. 